\title{
Cardiac magnetic resonance imaging in stable ischaemic heart disease
}

\author{
S. W. Kirschbaum • P. J. de Feyter • R.-J. M. van Geuns
}

Published online: 13 April 2011

(C) The Author(s) 2011. This article is published with open access at Springerlink.com

\begin{abstract}
Cardiac magnetic resonance imaging (CMR) is a new robust versatile non-invasive imaging technique that can detect global and regional myocardial dysfunction, presence of myocardial ischaemia and myocardial scar tissue in one imaging session without radiation, with superb spatial and temporal resolution, inherited three-dimensional data collection and with relatively safe contrast material. The reproducibility of CMR is high which makes it possible to use this technique for serial assessment to evaluate the effect of revascularisation therapy in patients with ischaemic heart disease.
\end{abstract}

Keywords Magnetic resonance imaging $\cdot$ Left ventricular function $\cdot$ Myocardial ischemia $\cdot$ Myocardial viability

\section{Introduction}

Ischaemic heart disease, despite advances of detection and treatment, remains the leading cause of morbidity and mortality in the Netherlands and more accurate detection and evaluation of coronary atherosclerosis may further reduce adverse outcome. Cardiac magnetic resonance imaging (CMR) is a new robust versatile noninvasive imaging technique that can detect global and regional myocardial dysfunction, presence of myocardial

S. W. Kirschbaum • P. J. de Feyter · R.-J. M. van Geuns $(\bowtie)$ Department of Cardiology, Erasmus University Medical Center, Thoraxcenter, room Ba 585, PO Box 2040, 3000 CA, Rotterdam, the Netherlands

e-mail: r.vangeuns@erasmusmc.nl ischaemia and myocardial scar tissue and has the advantage that it can evaluate all these parameters in one imaging session without the need for ionising radiation and with the use of relatively safe contrast material. CMR has demonstrated a high interstudy reproducibility which makes it possible to use this technique for serial assessment in an individual patient to evaluate the effect of treatment. The different diagnostic possibilities of CMR in patients with ischaemic heart disease will be discussed with the focus on the strengths and weaknesses of each particular feature.

\section{CMR for assessment of left ventricular function}

CMR is a precise and highly reproducible technique to assess left ventricular (LV) function, volume and mass with a lower intraobserver, interobserver, and interstudy variability $[1,2]$ as compared with LV angiography or echocardiography [3-5]. CMR is currently considered the standard of reference for these parameters and is frequently used as a surrogate endpoint in clinical trials $[6,7]$. These measurements are obtained using dynamic imaging where a series of parallel short-axis images is acquired covering the whole ventricle from the atrioventricular transition to the apex. The long-axis images of both the two- and four-chamber views in the end-diastolic phase at end-expiration provide the reference images to acquire a series of short-axis images (Fig. 1). To estimate LV functional volume and mass, the Simpson method is used where the volume of each individual slice is calculated and added [8]. Even though the Simpson's method has been the standard method of CMR LV analysis for many years, 
Fig. 1 The end-diastolic image of the two and four chamber image (upper part) is used to position a stack of short-axis images (bottom) from basal to apical with a slice thickness of $8 \mathrm{~mm}$
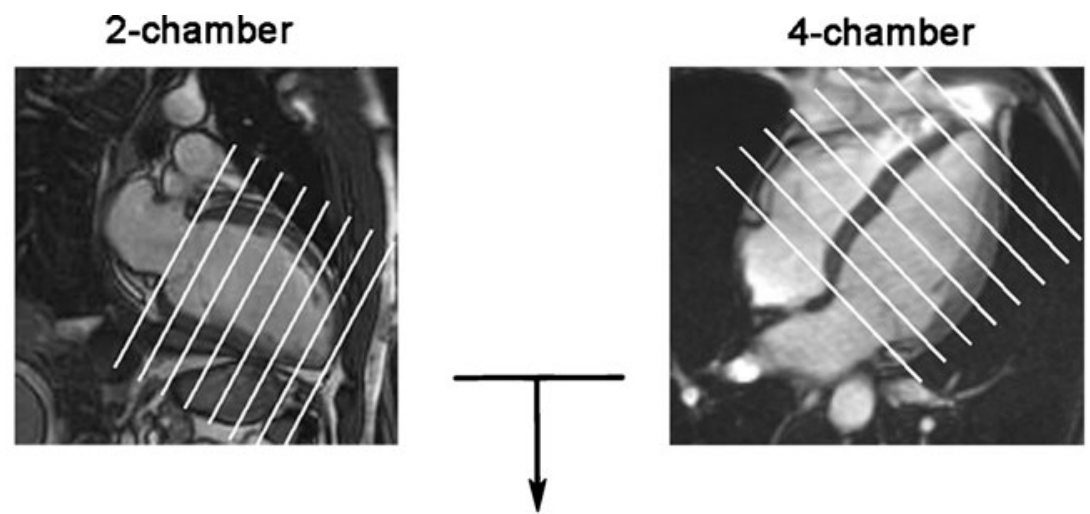

Short axis

\section{End diastolic phase}

Basal

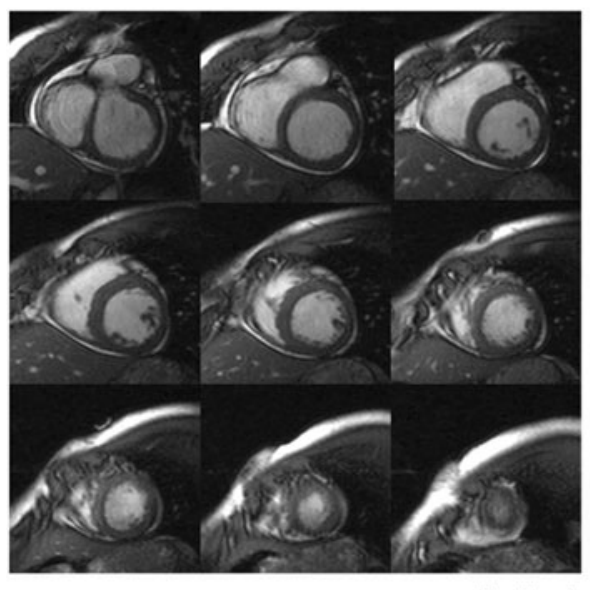

End systolic phase

Basal

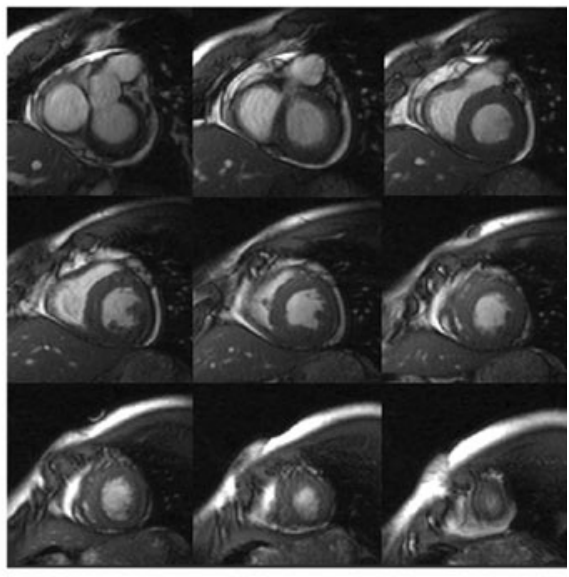

Apical variability in LV measurements is still present. Sources of variability are inconsistency in planning of the images during acquisition and post-procedure analysis, when the annalist has to indicate the most upper and lower border of the left ventricle. Integrating the contours of the long-axis to short-axis contours significantly reduces interstudy variability of all LV functional parameters and may be the preferred analysis method (Fig. 2) [1].

Using CMR the left ventricle can be studied in detail (Fig. 3) and even thin structures such as the heart valves and papillary muscles can be visualised. A regular heart rhythm and consistent breath holding are essential for highly detailed imaging as each dynamic loop is acquired during a 10-12 s period of suspended breathing. When patients cannot hold their breath or have an irregular heart rhythm, image quality may be poor and is often not good enough for accurate analysis due to respiratory motion or mistriggering of the ECG. In these cases real-time dynamic acquisition without breath holding can be performed and each image is acquired during a single heartbeat. A disadvantage of this technique is the lower spatial and temporal resolution which may hamper precise LV analysis [9].

\section{CMR for the detection of ischaemia}

If a treadmill electrocardiography test for the identification of ischaemia is physically impossible or the test is inconclusive, non-invasive perfusion imaging may be indicated. CMR is the latest addition to the spectrum of non-invasive myocardial perfusion or wall motion imaging modalities. The advantage of CMR over other technologies is its high resolution and contrast-to-noise ratio. The two main CMR methods for the assessment of myocardial ischaemia are high-dose dobutamine stress test with wall motion assessment and first pass myocardial perfusion under pharmacological stress with adenosine. The patient should be closely monitored during stress CMR by measuring blood pressure and heart rhythm during infusion of these pharmacological stressors. One must keep in mind that the ST-segment deviation is disturbed in the static 
Fig. 2 Illustration of the method using the information of the long axis to achieve left ventricular volumes mass and function. During the enddiastolic phase (a, c), slice 1 is partially included in the LV volume, slice 2 is totally included based on the long axis areas, while during systole (figure $\mathbf{b}, \mathbf{d}$ ) $0 \%$ is included of slice 1 and slice 2 is only partially included. At the apex, slice 3 is partially included for both phases (a, b, c and d) in LV functional assessment. By integrating the long axis and thereby more accurately indicating left ventricular borders the variability can be significantly reduced. Copyright Lippincott Williams \& Wilkins
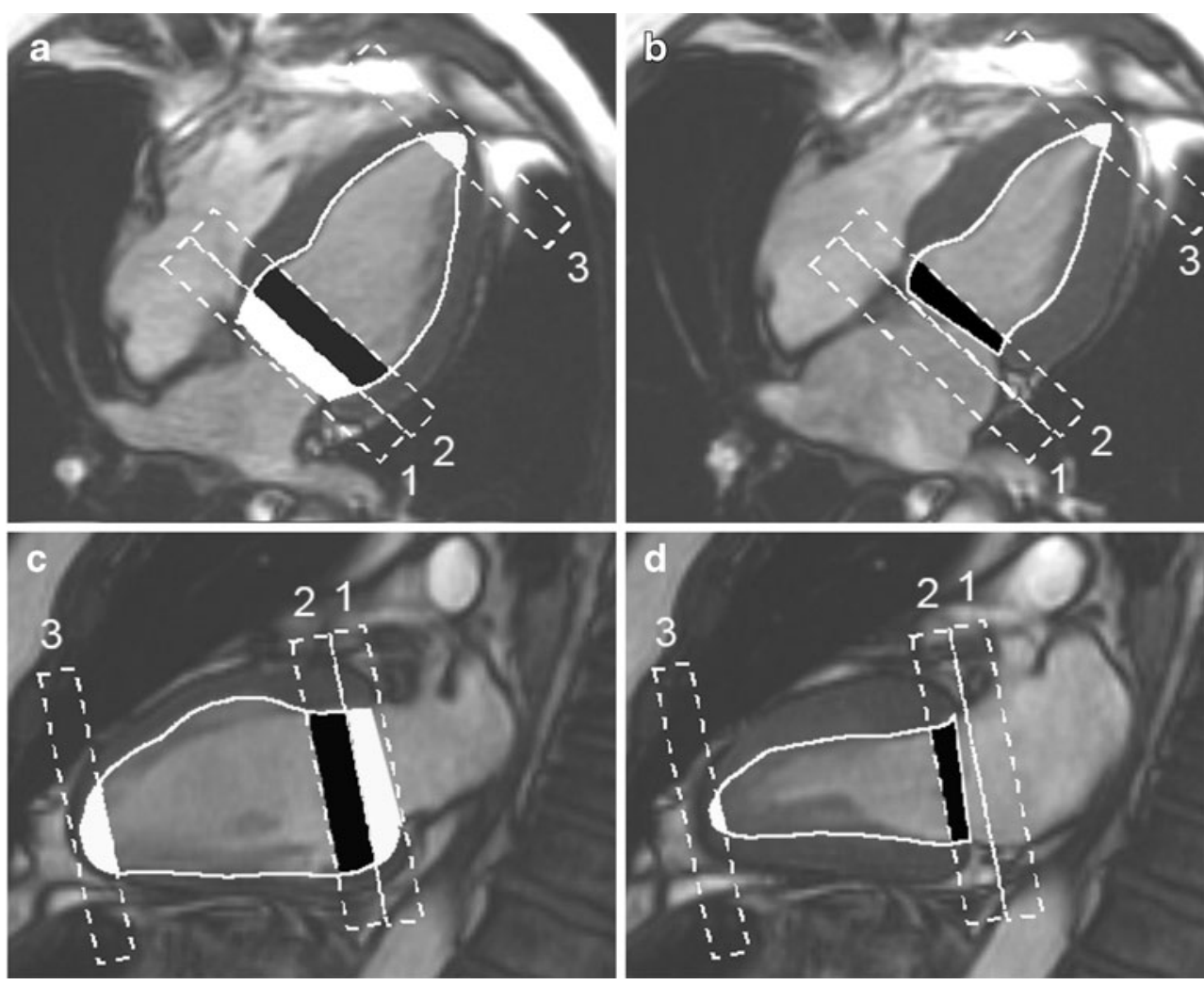

magnetic field and detection of ischaemia by ECG monitoring is not possible.

High-dose dobutamine stress with wall motion assessment

During dobutamine infusion, similar to echocardiography, functional imaging is used to detect the ischaemiainduced wall motion abnormalities at increasing doses from $10,20,30$ and $40 \mu \mathrm{g} / \mathrm{kg} / \mathrm{min}$ every $3 \mathrm{~min}$ at each stress level and imaging is repeated at every stress level in both long- and short-axis orientations. It is important to closely monitor the development of wall-thickening abnormalities and if these develop the test is considered positive for a haemodynamically significant coronary stenosis. In a meta analysis of the pooled data of 13 publications investigating 735 patients using dobutamine stress CMR for the detection of ischaemia, sensitivity was $83 \%(95 \%$ CI $79-88 \%)$ and specificity $86 \%(95 \%$ CI 81-91\%) when analysed on a per-patient level [10]. Nagel et al. compared dobutamine stress CMR with dobutamine stress echocardiography in patients with suspected coronary artery disease [11]. They found that dobutamine stress CMR had a higher diagnostic performance. The sensitivity increased from 74 to $86 \%$ and specificity increased from 70 to $86 \%$ (both $p<0.05$ ) using coronary angiography as the standard of reference. Also in
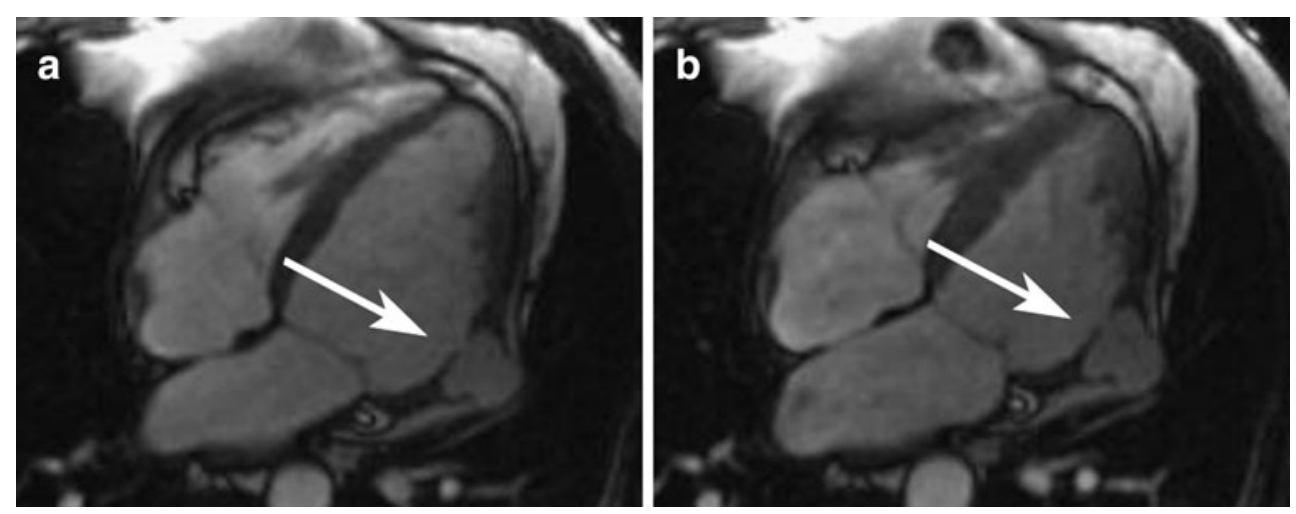

Fig. 3 This is a four-chamber view of a 64-year-old patient, with a recent history of a myocardial infarction, complicated by a pseudo aneurysm (arrow) of the anterolateral wall (end-diastolic phase, a; end-systolic phase, b) 
patients with a poor acoustic echo window, image quality of CMR was able to detect the presence of coronary artery disease with a sensitivity varying from 75 to $92 \%$ depending on the extent of coronary artery disease and a specificity of $83 \%$ using quantitative coronary angiography as standard of reference [12].

Although CMR is a relatively new technology, it has proven prognostic value; patients with inducible ischaemia were at higher risk of developing a myocardial infarction or cardiac death independent and incremental to the presence of conventional risk factors for coronary arteriosclerosis [13].

\section{Adenosine-stressed first-pass myocardial perfusion}

Myocardial perfusion imaging is based on the changes of myocardial signal intensity during the first passage of a contrast bolus of intravenously injected contrast agent into the myocardium at rest and during stress using adenosine as a pharmacological stressor. Flow limiting coronary stenosis during stress causes less perfusion of the subtended myocardium than myocardium that is supplied by normal vessels. This is visualised on CMR images as a darker myocardial area (less perfusion, less contrast) during stress as compared with normal perfused myocardial areas.

Perfusion of the myocardium can be assessed visually (perfusion present or absent) or quantitatively by calculating the myocardial perfusion reserve index [14].

The MR Impact trial [15], a multicentre multivendor prospective trial, compared perfusion CMR with single photon emission tomography (SPECT) and concluded that perfusion CMR in the entire study population was superior to SPECT. The superior sensitivity and specificity of stress perfusion CMR is most likely due to the better spatial resolution as compared with SPECT. In addition, SPECT is hampered by its radiation exposure and attenuation artifacts that sometimes make it difficult to correctly interpret the scans. Visual assessment of perfusion images, as in the MRImpact trial, by experienced readers has adequate diagnostic accuracy, but this simple approach is not always sufficient and subtle differences may be missed as might be needed for follow-up examination or to evaluate therapeutic effects on myocardial perfusion. Quantitative analysis is more objective; it also has the advantage that in patients with three-vessel disease, balanced perfusion defects can be detected.

To determine the myocardial perfusion reserve index for quantitative assessment, the relative upslope of a given segment during stress is divided by the relative upslope of a given segment at rest $[16,17]$. Relative upslope is defined as the maximum upslope of the signal intensity curve divided by the maximum upslope of the LV cavity curve.

A disadvantage of perfusion CMR is the fact that the investigation is cumbersome, time-consuming and acquisition and interpretation of images requires extensive expe- rience. In a meta analysis of 14 studies of 1183 patients, pooled data analysis showed a sensitivity of $91 \%$ (95\% CI 88-94) and a specificity of $81 \%(95 \%$ CI $77-85)$ for adenosine perfusion CMR on a per-patient level compared with coronary angiography as standard of reference [10]. Two more recent studies showed the same diagnostic accuracy (Table 1) [18, 19].

\section{CMR for the assessment of viability}

LV dysfunction may be the result of viable or non-viable dysfunctional myocardium. The latter may not recover after revascularisation therapy while viable myocardium in patients with chronic ischaemic heart disease will recover in weeks, months or years after revascularisation. Viable myocardium is associated with repetitive transient ischaemia or persistently reduced myocardial blood flow, so-called hibernating myocardium, which must be distinguished from permanently damaged infarcted myocardium. Viable dysfunctional myocardium is defined as recovery of regional myocardial dysfunction after revascularisation. CMR viability assessment is achieved using low-dose dobutamine as a functional response to examine contractile reserve or late gadolinium enhancement as necrosis imaging or simply assessing end-diastolic wall thickness.

End-diastolic wall thickness was the first parameter to be used as a viability parameter prior to late gadolinium enhancement and an end-diastolic wall thickness of $<5.5 \mathrm{~mm}$ represented non-viable tissue [20-22].

\section{Low-dose dobutamine}

Dobutamine is known to stimulate the $\beta$-receptors of the myocytes, increases the ATP and oxygen consumption and therefore improves contraction. The presence of contractile reserve during low-dose dobutamine, which is tested at two different doses (5 and $10 \mu \mathrm{g} / \mathrm{kg} / \mathrm{min}$ ), has a higher predictive accuracy as compared with the end-diastolic wall thickness (91 vs. $79 \%$ for preserved wall thickness) [23]. With current fast CMR techniques, one is able to scan the left ventricle at both stress levels and a useful approach

Table 1 Sensitivity and specificity of perfusion MRI

\begin{tabular}{llll}
\hline Author [refH] & N & Sensitivity (\%) & Specificity (\%) \\
\hline Gebker [19] & 455 & 91 & 70 \\
Klem [18] & 136 & 84 & 88 \\
Nandalur [10] & 1183 & 91 & 81 \\
Schwitter [15] & 234 & 85 & 67 \\
\hline
\end{tabular}

$N=$ number of patients 
Fig. 4 A three-chamber view in the end-diastolic phase (a) and the end-systolic phase (b) at rest of a patient with an old anterior wall infarction and a chronic total coronary occlusion of the left anterior descending artery, and a three-chamber view of the same patient after administration of low-dose dobutamine in the end-diastolic phase (c) and the end-systolic phase (d) where an improvement in contractility was detected in the anteroseptal wall. LA, left atrium; A, aorta; $\mathrm{LV}$, left ventricle
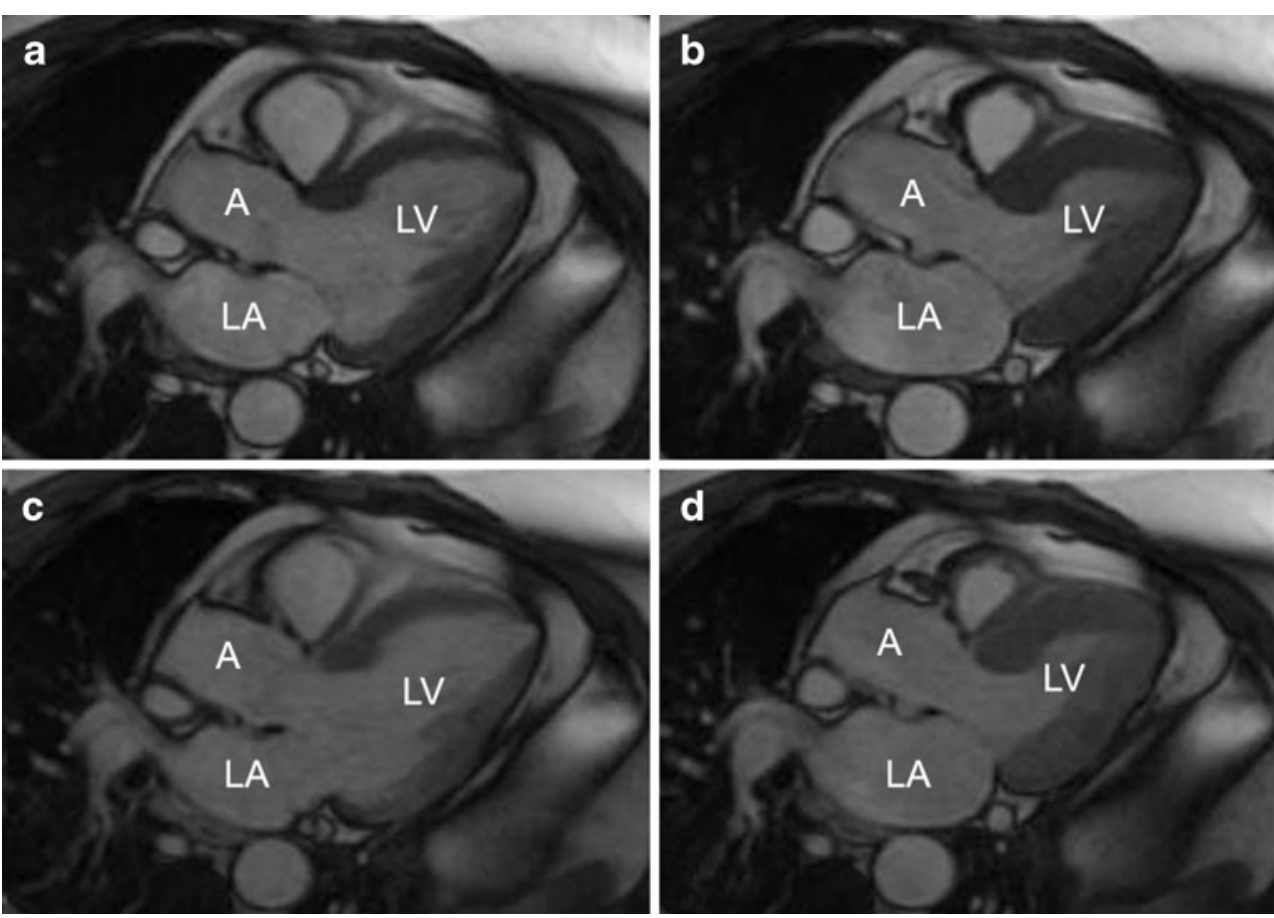

is to acquire three short-axis views and three long-axis views within four breath holds during each stress level. An example of a patient with contractile reserve during dobutamine in a region perfused by a chronic total occlusion is presented in Fig. 4.

\section{CMR late gadolinium enhancement}

Contrast late enhancement CMR allows visualisation of the distribution of myocardial scarring in post-infarction patients [24-29]. Kim et al. [24] were the first to show that delayed enhancement strongly predicts functional improvement after revascularisation. In segments with no infarction the likelihood of recovery was high, $78 \%$ of the segments recovered after revascularisation while only one segment with $>75 \%$ infarct transmurality improved.

Delayed enhancement images are acquired 10-20 min after administration of a gadolinium-based contrast agent. The resulting image will show normal myocardium as black and infarcted myocardium as white (Fig. 5). In acute myocardial infarction, the contrast agent passively diffuses into the intracellular space because the membrane is ruptured while chronic myocardial infarction contains collagenous material replacing the myocytes which increases the extracellular space causing increased contrast concentration and thus enhancement on delayed imaging CMR [30]. Gadolinium itself is toxic but when chelated to other molecules can be safely administered to most patients.

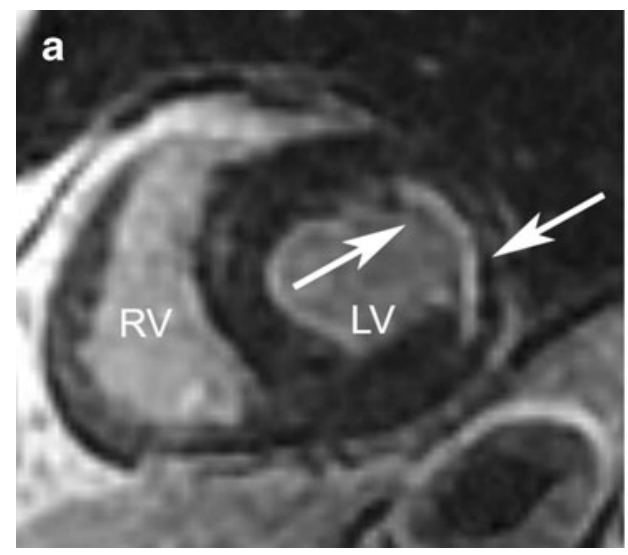

Fig. 5 Short-axis delayed enhancement image (a) with a subendocardial infarct in the lateral wall between the white arrows. A twochamber delayed enhancement image with a transmural infarction of

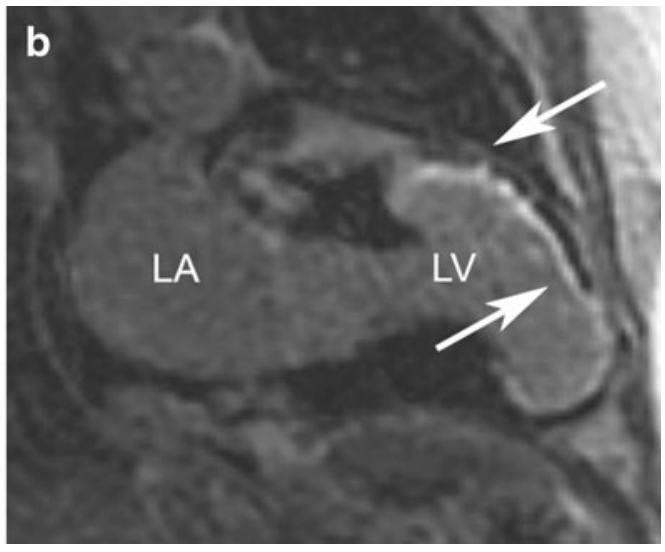

the anterior wall between arrows (b). RV, right ventricle; LV, left ventricle; LA, left atrium 
However, in patients with severe kidney disease gadoliniumbased contrast agents may increase the risk of developing a rare and potentially fatal condition known as nephrogenic systemic fibrosis. It involves the formation of excess fibrous connective tissue in the skin, eyes, joints and body organs. Symptoms may include stiffness, hardening and tightening of the skin. If body organs are involved it eventually leads to death. The Federation of Food and Drug Administration (FDA) advises not to use this agent in patients with acute kidney injury or chronic, severe kidney disease (with a glomerular filtration rate $<30 \mathrm{ml} / \mathrm{min} / 1.73 \mathrm{~m}^{2}$ ).

\section{When to assess functional improvement of viable myocardium after revascularisation?}

Initial studies examining the functional recovery of revascularised viable myocardium were performed at 6 months after revascularisation. It was assumed that this time period was sufficient for the processes underlying restoration of dysfunctional myocardium. However, we hypothesised that it may require longer for recovery and we examined patients at 6 months and again at 3 years of follow-up [31]. Improvement in segments with a small subendocardial infarction (a transmural extent of infarction of $<25 \%$ ) was observed at 6 months and further improvement occurred at 3-year follow-up. In segments with more extensive scarring, a transmural extent of infarction between 25$75 \%$, improvement was only observed at 3 -year follow-up but not at 6 months.

\section{Non-ischaemic heart disease}

Abnormal enhancement may also be present in patients with other myocardial diseases such as myocarditis, infiltrative cardiomyopathy such as amyloidosis or Fabry's disease, hypertrophic cardiomyopathy or tumours. The enhancement pattern in these patients is different from the enhancement pattern seen in patients with a myocardial infarction, where it usually involves the endocardium and may extend to the epicardium and is linked with a specific coronary vessel territory. In patients with non-ischaemic heart disease, enhancement patterns are different and vary between mid-myocardial enhancement, a more patchy multifocal distribution or epicardial enhancement. Myocardial contraction may also be impaired in these regions [30].

\section{Conclusion}

CMR is considered to be the standard of reference for the comprehensive assessment of the left ventricle that can measure cardiac function, detect ischaemia and has the ability to assess viable myocardium in one imaging session without the use of ionising radiation. However, in patients with stable anginal symptoms, anatomical information of the coronary vessel is also required for clinical decisionmaking. CMR falls short for adequately visualising the coronary artery and improvements are highly desirable to achieve complimentary anatomical and functional information in one investigational session.

Open Access This article is distributed under the terms of the Creative Commons Attribution Noncommercial License which permits any noncommercial use, distribution, and reproduction in any medium, provided the original author(s) and source are credited.

\section{References}

1. Kirschbaum SW, Baks T, Gronenschild EH, et al. Addition of the long-axis information to short-axis contours reduces interstudy variability of left-ventricular analysis in cardiac magnetic resonance studies. Invest Radiol. 2008;43:1-6.

2. Bellenger NG, Davies LC, Francis JM, et al. Reduction in sample size for studies of remodeling in heart failure by the use of cardiovascular magnetic resonance. J Cardiovasc Magn Reson. 2000;2:271-8.

3. Danilouchkine MG, Westenberg JJ, de Roos A, et al. Operator induced variability in cardiovascular MR: left ventricular measurements and their reproducibility. J Cardiovasc Magn Reson. 2005;7:447-57.

4. Bogaert JG, Bosmans HT, Rademakers FE, et al. Left ventricular quantification with breath-hold MR imaging: comparison with echocardiography. Magma. 1995;3:5-12.

5. Grothues F, Smith GC, Moon JC, et al. Comparison of interstudy reproducibility of cardiovascular magnetic resonance with twodimensional echocardiography in normal subjects and in patients with heart failure or left ventricular hypertrophy. Am J Cardiol. 2002;90:29-34.

6. Schachinger V, Assmus B, Britten MB, et al. Transplantation of progenitor cells and regeneration enhancement in acute myocardial infarction: final one-year results of the TOPCARE-AMI Trial. J Am Coll Cardiol. 2004;44:1690-9.

7. Meyer GP, Wollert KC, Lotz J, et al. Intracoronary bone marrow cell transfer after myocardial infarction: 18 months' follow-up data from the randomized, controlled BOOST (BOne marrOw transfer to enhance ST-elevation infarct regeneration) trial. Circulation. 2006;113:1287-94.

8. Dulce MC, Mostbeck GH, Friese KK, et al. Quantification of the left ventricular volumes and function with cine MR imaging: comparison of geometric models with three-dimensional data. Radiology. 1993;188:371-6.

9. Setser RM, Fischer SE, Lorenz CH. Quantification of left ventricular function with magnetic resonance images acquired in real time. J Magn Reson Imaging. 2000;12:430-8.

10. Nandalur KR, Dwamena BA, Choudhri AF, et al. Diagnostic performance of stress cardiac magnetic resonance imaging in the detection of coronary artery disease: a meta-analysis. J Am Coll Cardiol. 2007;50:1343-53.

11. Nagel E, Lehmkuhl HB, Bocksch W, et al. Noninvasive diagnosis of ischemia-induced wall motion abnormalities with 
the use of high-dose dobutamine stress MRI: comparison with dobutamine stress echocardiography. Circulation. 1999;99:76370.

12. Hundley WG, Hamilton CA, Thomas MS, et al. Utility of fast cine magnetic resonance imaging and display for the detection of myocardial ischemia in patients not well suited for second harmonic stress echocardiography. Circulation. 1999;100:1697-702.

13. Hundley WG, Morgan TM, Neagle CM, et al. Magnetic resonance imaging determination of cardiac prognosis. Circulation. 2002;106:2328-33.

14. Wilke N, Jerosch-Herold M, Wang Y, et al. Myocardial perfusion reserve: assessment with multisection, quantitative, first-pass MR imaging. Radiology. 1997;204:373-84.

15. Schwitter J, Wacker CM, van Rossum AC, et al. MR-IMPACT: comparison of perfusion-cardiac magnetic resonance with singlephoton emission computed tomography for the detection of coronary artery disease in a multicentre, multivendor, randomized trial. Eur Heart J. 2008;29:480-9.

16. Al-Saadi N, Nagel E, Gross M, et al. Noninvasive detection of myocardial ischemia from perfusion reserve based on cardiovascular magnetic resonance. Circulation. 2000;101:1379-83.

17. Jerosch-Herold M, Seethamraju RT, Swingen CM, et al. Analysis of myocardial perfusion MRI. J Magn Reson Imaging. 2004;19:758-70.

18. Klem I, Greulich S, Heitner JF, et al. Value of cardiovascular magnetic resonance stress perfusion testing for the detection of coronary artery disease in women. JACC. 2008;1:436-45.

19. Gebker R, Jahnke C, Manka R, et al. Additional value of myocardial perfusion imaging during dobutamine stress magnetic resonance for the assessment of coronary artery disease. Circ Cardiovasc Imaging. 2008;1:122-30.

20. Baer FM, Voth E, Schneider CA, et al. Comparison of low-dose dobutamine-gradient-echo magnetic resonance imaging and positron emission tomography with $[18 \mathrm{~F}]$ fluorodeoxyglucose in patients with chronic coronary artery disease. A functional and morphological approach to the detection of residual myocardial viability. Circulation. 1995;91:1006-15.

21. Baer FM, Smolarz K, Theissen P, et al. Regional 99mTcmethoxyisobutyl-isonitrile-uptake at rest in patients with myocardial infarcts: comparison with morphological and functional parameters obtained from gradient-echo magnetic resonance imaging. Eur Heart J. 1994;15:97-107.

22. Baer FM, Smolarz K, Jungehulsing $M$, et al. Chronic myocardial infarction: assessment of morphology, function, and perfusion by gradient echo magnetic resonance imaging and $99 \mathrm{mTc}-\mathrm{methoxyisobutyl-isonitrile} \mathrm{SPECT}$. Am Heart J. 1992;123:636-45.

23. Baer FM, Theissen P, Schneider CA, et al. Dobutamine magnetic resonance imaging predicts contractile recovery of chronically dysfunctional myocardium after successful revascularization. J Am Coll Cardiol. 1998;31:1040-8.

24. Kim RJ, Wu E, Rafael A, et al. The use of contrast-enhanced magnetic resonance imaging to identify reversible myocardial dysfunction. New Engl J Med. 2000;343:1445-53.

25. Kim RJ, Fieno DS, Parrish TB, et al. Relationship of MRI delayed contrast enhancement to irreversible injury, infarct age, and contractile function. Circulation. 1999;100:1992-2002.

26. Wu E, Judd RM, Vargas JD, et al. Visualisation of presence, location, and transmural extent of healed Q-wave and non-Q-wave myocardial infarction. Lancet. 2001;357:21-8.

27. Wagner A, Mahrholdt H, Holly TA, et al. Contrast-enhanced MRI and routine single photon emission computed tomography (SPECT) perfusion imaging for detection of subendocardial myocardial infarcts: an imaging study. Lancet. 2003;361:374-9.

28. Judd RM, Lugo-Olivieri CH, Arai M, et al. Physiological basis of myocardial contrast enhancement in fast magnetic resonance images of 2-day-old reperfused canine infarcts. Circulation. 1995;92:1902-10.

29. Baks T, van Geuns RJ, Duncker DJ, et al. Prediction of left ventricular function after drug-eluting stent implantation for chronic total coronary occlusions. J Am Coll Cardiol. 2006;47:721-5.

30. Mahrholdt H, Wagner A, Judd RM, et al. Delayed enhancement cardiovascular magnetic resonance assessment of non-ischaemic cardiomyopathies. Eur Heart J. 2005;26:1461-74.

31. Kirschbaum SW, Baks T, van den Ent M, et al. Evaluation of left ventricular function three years after percutaneous recanalization of chronic total coronary occlusions. Am J Cardiol. 2008;101:179-85. 